\title{
Periodicity of the Greatest Common Divisors within Generalized Divisibility Sequences
}

\author{
Ivica Martinjak \\ Faculty of Science, University of Zagreb \\ Bijenička cesta 32, HR-10000 Zagreb, Croatia \\ and \\ Sanja Tipurić - Spužević \\ University of Mostar, Faculty of Science and Education, \\ Matice Hrvatske b.b., 88000 Mostar, Bosnia and Herzegovina
}

\begin{abstract}
This paper addresses divisibility properties of some families of sequences arising from partial sums of a strong divisibility sequence. In particular, we demonstrate periodicity of greatest common divisor within 1-fibonacci numbers. We also present congruences within this sequence modulo a prime number $p$ where $p \equiv \pm 1(\bmod 5)$ and $p \equiv \pm 2(\bmod 5)$.
\end{abstract}

Keywords: divisibility sequence, elliptic divisibility sequences, hypersequence, Fibonacci numbers, Mersenne numbers, Somos sequence, elliptic curves

MSC: 11A05, 11B39

\section{Introduction}

A divisibility sequence is an integer sequence $\left(d_{n}\right)_{n \geq 0}$ with the property that an index $n$ being a multiple of index $m$ imply the term $\bar{d}_{n}$ is a multiple of $d_{m}$,

$$
m\left|n \Longrightarrow d_{m}\right| d_{n}
$$

for all natural numbers $m, n$. If for the sequence $\left(d_{n}\right)_{n \geq 0}$ we have

$$
\operatorname{gcd}\left(d_{m}, d_{n}\right)=d_{\operatorname{gcd}(m, n)},
$$

DOI: https://doi.org/10.5592/CO/CCD.2018.07 
then such sequence is called strong divisibility sequence. It is immediately seen that a strong divisibility sequence is also divisibility sequence.

An important class of these sequences are elliptic divisibility sequences (EDS). An elliptic divisibility sequence is a sequence of integers $(W n)_{n \geq 0}$ satisfying the recursive relation

$$
W_{n+m} W_{n-m} W_{1}^{2}=W_{n+1} W_{n-1} W_{m}^{2}-W_{m+1} W_{m-1} W_{n}^{2}
$$

and such that $W_{n}$ divides $W_{m}$ whenever $n$ divides $m$. It is known that if the initial conditions for (3) satisfies

i) $W_{1}=1$,

ii) $W_{2}, W_{3}, W_{4} / W_{2} \in \mathbb{Z} \backslash 0$

then $W_{n}$ is an integer for every $n$. As a further basic property of EDS we have that if the sequence $\left(W_{n}\right)_{n \geq 0}$ is a solution of (3) then we have

$$
\begin{aligned}
W_{2 n+1} & =W_{n+2} W_{n}^{3}-W_{n-1} W_{n+1}^{3}, \quad n \geq 1 \\
W_{2 n} W_{2} & =W_{n}\left(W_{n+2} W_{n-1}^{2}-W_{n-2} W_{n+1}^{2}, \quad n \geq 2 .\right.
\end{aligned}
$$

An example of such sequences of numbers is the sequence

$$
1,1,1,-1,-2,-3,-1,7,11,20,-19,-87,-191,-197,1018, \ldots
$$

(the sequence A050512 in the OEIS). As another example let as mention the sequence $\left(G_{n}\right)_{n \geq 0}$ consisting of every second Fibonacci numbers is a EDS, $G_{n}=F_{2 m}$. We let $\left(h_{n}\right)_{h \geq 0}$ denote the sequence defined by $h_{n}=(n / 3)$ where $n \in \mathbb{N}$ and $(a / p)$ denote the Legendre symbol, for the prime number $p$.

Among notable representatives of divisibility sequences we have Mersenne numbers defined by the explicit formula

$$
M_{n}=2^{n}-1, \quad n \geq 0,
$$

as well as the Fibonacci numbers,

$$
F_{n}=\frac{1}{\sqrt{5}}\left[\left(\frac{1+\sqrt{5}}{2}\right)^{n}-\left(\frac{1-\sqrt{5}}{2}\right)^{n}\right]
$$

Both of these sequences appears in various number theoretical and combinatorial context. In addition to certain families of Dyck paths, the $n$-th Mersenne number 
appears as the number of nonempty subsets of a set with $n$ elements, as a $q$-binomial coefficient, a rank of matroids, etc. (the sequence A000225 in the OEIS). Recall that Fibonacci numbers appears as the solutions of the Diophantine equation

$$
x^{2}-5 y^{2}=4(-1)^{n},
$$

i.e. we have the Fibonacci sequence $\left(F_{n}\right)_{n \geq 0}$ and the Lucas sequence $\left(L_{n}\right)_{n \geq 0}$ as the solutions $(x, y)=\left(L_{n}, F_{n}\right)$ of $(4)$, where the Lucas numbers are defined by the same recurrence relation as the Fibonacci numbers but with the initial conditions $L_{0}=2, L_{1}=1$. One can also use the Diophantine equation (4) as a definition of these two sequences of numbers.

This work aim at finding divisibility properties of some families of generalized divisibility sequences. We were curious to establish how the properties (1) and (2) are inherited within such sequences.

\section{Previous results and motivation}

A complete characterization of divisibility sequences arising from linear recurrences is done by Bézivin, Pethő, and van der Poorten [2]. Recent development is done by Ingram [11], Silverman [18] and Gezer and Bizim [6]. There are further generalizations and extensions of this notion. A natural generalization of divisibility sequences is through divisibility of ideals in a ring. One can find more on this in a work of Silverman [17]. Results on matrix divisibility sequences (a sequence of matrices with properties analogue to (1)) are found by Cornelissen and Reynolds [4] as well as Górnisiewicz [10]. Among other classes of divisibility sequences, let mention a class of sequences defined as

$$
d_{n}(\alpha)=\max \left\{d \in \mathbb{Z}: \alpha^{n} \equiv 1 \quad(\bmod d)\right\}
$$

where $\alpha$ is an algebraic integer. Moreover, such sequences also satisfy property (2) i.e. they are strong divisibility sequences, which is proved by Silverman [16].

Among many remarkable properties of the Fibonacci sequence $\left(F_{n}\right)_{n \geq 0}, F_{n+2}=$ $F_{n+1}+F_{n}, F_{0}=0, F_{1}=1$ we have that when $m$ divide $n$, then $F_{m}$ divide $F_{n}$,

$$
m\left|n \Longrightarrow F_{m}\right| F_{n} \text {. }
$$

There is also identity

$$
F_{m+n}=F_{m+1} F_{n}+F_{m} F_{n-1}
$$


and as a consequence of these two facts one can derive that the greatest common divisor of the Fibonacci numbers $F_{m}$ and $F_{n}$ is again Fibonacci number, that one whose index is $\operatorname{gcd}(m, n)$,

$$
\operatorname{gcd}\left(F_{m}, F_{n}\right)=F_{\operatorname{gcd}(m, n)} .
$$

Details on proof of this one can find in [1]. Among further divisibility properties of Fibonacci numbers is a well known fact that

$$
\begin{aligned}
F_{p} & \equiv\left(\frac{p}{5}\right) \quad(\bmod p) \\
F_{p \pm 1} & \equiv \frac{1 \pm\left(\frac{p}{5}\right)}{2}(\bmod p)
\end{aligned}
$$

where $p$ is an odd prime.

In what follows we present divisibility properties of the sequences arising from partial sums of a family of strong divisibility sequence. In particular, the hyperfibonacci sequence of the $r$ th generation $\left(F_{n}^{(r)}\right)_{n \geq 0}$, is defined by the recurrence relation

$$
F_{n}^{(r)}=\sum_{k=0}^{n} F_{k}^{(r-1)}, \quad F_{n}^{(0)}=F_{n}, \quad F_{0}^{(r)}=0, \quad F_{1}^{(r)}=1,
$$

where $r \in \mathbb{N}$ and $F_{n}$ is the $n$th term of the Fibonacci sequence $\left(F_{n}\right)_{n \geq 0}$. These sequences are introduced by Dil and Mezö, in a study of a symmetric algorithm for hyperharmonic, Fibonacci and some other integer sequences [8]. Several number theoretical, combinatorial and algebraical properties of hyperfibonacci sequences is already known $[3,13,14,20]$. An alternative definition of $\left(F_{n}^{(r)}\right)_{n \geq 0}$ is by the recurrence relation

$$
F_{n+2}^{(r)}=F_{n+1}^{(r)}+F_{n}^{(r)}+\left(\begin{array}{c}
n+r \\
r-1
\end{array}\right), n \geq 0
$$

where initial values are $F_{0}^{(r)}=0, F_{1}^{(r)}=1$. The proof of this one can find in [5]. For $r=1$ this relation gives the sequence of numbers

$$
0,1,2,4,7,12,20,33,54,88,143,232,376, \ldots,
$$

for $r=2$ we have the sequence

$$
0,1,3,7,14,26,46,79,133,221,364,596,972, \ldots
$$


etc. When $r=1$, relation (10) reduces to

$$
F_{n+2}^{(1)}=F_{n+1}^{(1)}+F_{n}^{(1)}+1 .
$$

We shall present divisibility properties of hypefibonacci numbers of the first generation $\left(F_{n}^{(1)}\right)_{n \geq 0}$. Throughout the paper, the hyperfibonacci sequence of the 1st generation we shall also call hyperfibonacci sequence, in short.

\section{The main result}

Every two consecutive Fibonacci numbers are relatively prime. In Lemma 1 we generalize this property on the case of hyperfibonacci numbers.

Lemma 1. Every three consecutive hyperfibonacci numbers $F_{n}^{(1)}, F_{n+1}^{(1)}, F_{n+2}^{(1)}, n \geq 0$ are relatively prime,

$$
\operatorname{gcd}\left(F_{n}^{(1)}, F_{n+1}^{(1)}, F_{n+2}^{(1)}\right)=1 .
$$

Proof. Using basic properties of the gcd function and the recurrence relation (11) we obtain

$$
\begin{aligned}
\operatorname{gcd}\left(F_{n}^{(1)}, F_{n+1}^{(1)}, F_{n+2}^{(1)}\right) & =\operatorname{gcd}\left(F_{n}^{(1)}, \operatorname{gcd}\left(F_{n+1}^{(1)}, F_{n+1}^{(1)}+F_{n}^{(1)}+1\right)\right) \\
& =\operatorname{gcd}\left(\operatorname{gcd}\left(F_{n}^{(1)}, F_{n}^{(1)}+1\right), F_{n+1}^{(1)}\right) \\
& =\operatorname{gcd}\left(1, F_{n}^{(1)}, F_{n+1}^{(1)}\right)=1 .
\end{aligned}
$$

In a similar fashion one can prove that every four consecutive hyperfibonacci numbers of the 2-nd generation are relatively prime. Furthermore, for $r \geq 3$ one can use the obvious equality for binomial coefficients

$$
\left(\begin{array}{c}
n+q \\
p
\end{array}\right)-\left(\begin{array}{c}
n+q-1 \\
p
\end{array}\right)=\left(\begin{array}{c}
n+q-1 \\
p-1
\end{array}\right)
$$


when applying Euclid algorithm. In particular, for $r=3$ we have

$$
\begin{aligned}
& \operatorname{gcd}\left(F_{n}^{(3)}, F_{n+1}^{(3)}, \ldots, F_{n+4}^{(3)}\right) \\
= & \operatorname{gcd}\left(F_{n}^{(3)}, \ldots, F_{n+3}^{(3)}, F_{n+3}^{(3)}+F_{n+2}^{(3)}+\left(\begin{array}{c}
n+5 \\
2
\end{array}\right)\right) \\
= & \operatorname{gcd}\left(F_{n}^{(3)}, \ldots, F_{n+3}^{(3)},\left(\begin{array}{c}
n+5 \\
2
\end{array}\right)\right) \\
= & \operatorname{gcd}\left(F_{n}^{(3)}, \ldots, F_{n+2}^{(3)}+F_{n+1}^{(3)}+\left(\begin{array}{c}
n+4 \\
2
\end{array}\right),\left(\begin{array}{c}
n+5 \\
2
\end{array}\right)\right) \\
= & \operatorname{gcd}\left(F_{n}^{(3)}, F_{n+1}^{(3)}, F_{n+2}^{(3)},\left(\begin{array}{c}
n+4 \\
2
\end{array}\right),\left(\begin{array}{c}
n+5 \\
2
\end{array}\right)\right) \\
= & \operatorname{gcd}\left(F_{n}^{(3)}, F_{n+1}^{(3)},\left(\begin{array}{c}
n+3 \\
2
\end{array}\right),\left(\begin{array}{c}
n+4 \\
2
\end{array}\right),\left(\begin{array}{c}
n+5 \\
2
\end{array}\right)\right) \\
= & \operatorname{gcd}\left(F_{n}^{(3)}, F_{n+1}^{(3)},\left(\begin{array}{c}
n+3 \\
2
\end{array}\right), n+4,1\right) \\
= & 1 .
\end{aligned}
$$

We formalize these arguments in the proof of the following Theorem 1.

Theorem 1. For $n \geq 0$, every $(r+2)$-tuple of consecutive hyperfibonacci numbers of $r$-th generation are relatively prime,

$$
\operatorname{gcd}\left(F_{n}^{(r)}, F_{n+1}^{(r)}, \ldots, F_{n+r+1}^{(r)}\right)=1 .
$$

Proof. When applying basic properties of the gcd function we use relations (11) and (13) to get

$$
\begin{aligned}
& \operatorname{gcd}\left(F_{n}^{(r)}, F_{n+1}^{(r)}, \ldots, F_{n+r+1}^{(r)}\right) \\
& =\operatorname{gcd}\left(F_{n}^{(r)}, F_{n+1}^{(r)},\left(\begin{array}{c}
n+r \\
r-1
\end{array}\right),\left(\begin{array}{c}
n+r+1 \\
r-1
\end{array}\right), \ldots,\left(\begin{array}{c}
n+2 r-1 \\
r-1
\end{array}\right)\right) \\
& =\operatorname{gcd}\left(F_{n}^{(r)}, F_{n+1}^{(r)},\left(\begin{array}{c}
n+r \\
r-1
\end{array}\right),\left(\begin{array}{c}
n+r \\
r-2
\end{array}\right), \ldots,\left(\begin{array}{c}
n+2 r-2 \\
r-2
\end{array}\right)\right) \\
& =\operatorname{gcd}\left(F_{n}^{(r)}, F_{n+1}^{(r)},\left(\begin{array}{c}
n+r \\
r-1
\end{array}\right),\left(\begin{array}{c}
n+r \\
r-2
\end{array}\right), \ldots, n+r, n+r+1\right) \\
& =\operatorname{gcd}\left(F_{n}^{(r)}, F_{n+1}^{(r)},\left(\begin{array}{c}
n+r \\
r-1
\end{array}\right),\left(\begin{array}{c}
n+r \\
r-2
\end{array}\right), \ldots, n+r, 1\right)=1 .
\end{aligned}
$$


In addition, we have that the greatest common divisor of some pairs of hyperfibonacci numbers is a Fibonacci number, as stated in Theorem 2.

Theorem 2. For $m, n \in \mathbb{N}$ the greatest common divisor of the $(4 m-3)$-th and $(4 m-1)$-st hyperfibonacci numbers is equal to $F_{2 m}$,

$$
\operatorname{gcd}\left(F_{4 m-3}^{(1)}, F_{4 m-1}^{(1)}\right)=F_{2 m} .
$$

Proof. Using the fact that the gcd of two numbers does not change if the largest number is replaced by its difference with the smaller one and applying the recurrence relation (11) we obtain

$$
\operatorname{gcd}\left(F_{4 m-3}^{(1)}, F_{4 m-1}^{(1)}\right)=\operatorname{gcd}\left(F_{4 m-3}^{(1)}, F_{4 m-2}^{(1)}+1\right)=\operatorname{gcd}\left(F_{4 m-5}^{(1)}-1, F_{4 m-4}^{(1)}+2\right) .
$$

When we continue to diminish the larger number this way, resulting number is always represented as a sum of a hyperfibonacci number and an integer, $F_{4 m-q}+a_{q}$ and $F_{m-q+1}+a_{q-1}$. According to the initial terms $a_{3}=0$ and $a_{2}=1$, the absolute value of the $n$-th number in sequence of these integers differentiate from the $n$-th Fibonacci number for 1 . More precisely, we have

$$
\begin{aligned}
& \operatorname{gcd}\left(F_{4 m-3}^{(1)}, F_{4 m-1}^{(1)}\right) \\
= & \operatorname{gcd}\left(F_{4 m-q}^{(1)}+(-1)^{q}\left(F_{q-2}+1\right), F_{4 m-q+1}^{(1)}+(-1)^{q+1}\left(F_{q-3}+1\right)\right)
\end{aligned}
$$

where $3 \leq q \leq 4 m$. Now, according to this fact we obtain

$$
\begin{aligned}
& \operatorname{gcd}\left(F_{4 m-3}^{(1)}, F_{4 m-1}^{(1)}\right)=\operatorname{gcd}\left(F_{4 m-3}^{(1)}-\left(F_{1}-1\right), F_{4 m-2}^{(1)}+\left(F_{0}+1\right)\right) \\
= & \operatorname{gcd}\left(F_{4 m-4}^{(1)}+\left(F_{2}+1\right), F_{4 m-3}^{(1)}-\left(F_{1}-1\right)\right) \\
= & \operatorname{gcd}\left(F_{2 m-2}^{(1)}+\left(F_{2 m}+1\right), F_{2 m-1}^{(1)}-\left(F_{2 m-1}-1\right)\right) \\
= & \operatorname{gcd}\left(2 F_{2 m}, F_{2 m+1}-F_{2 m-1}\right)=\operatorname{gcd}\left(2 F_{2 m}, F_{2 m}\right)=F_{2 m},
\end{aligned}
$$

which completes the proof.

For an alternative proof of Theorem 2 we have the following. By the product expansion formula we have

$$
F_{m+n}=F_{m} L_{n}+(-1)^{n+1} F_{m-n}
$$


and from it we get

$$
F_{4 m}=F_{2 m} L_{2 m} \text { and } F_{4 m-1}=F_{2 m} L_{2 m-1}+(-1)^{2 m} F_{1}=F_{2 m} L_{2 m-1}+1
$$

We now have:

$$
\begin{aligned}
\operatorname{gcd}\left(F_{4 m-3}^{(1)}, F_{4 m-1}^{(1)}\right) & =\operatorname{gcd}\left(F_{4 m-1}-1, F_{4 m+1}-1\right)=\operatorname{gcd}\left(F_{4 m-1}-1, F_{4 m}\right)= \\
& =\operatorname{gcd}\left(F_{2 m} L_{2 m-1}, F_{2 m} L_{2 m}\right)=F_{2 m} \operatorname{gcd}\left(L_{2 m-1}, L_{2 m}\right)=F_{2 m}
\end{aligned}
$$

As an example, let consider the case when $m=3$. According to Theorem 2 the greatest common divisor of numbers $F_{9}^{(1)}(=88)$ and $F_{11}^{(1)}(=232)$ is equal to

$$
\begin{aligned}
& \operatorname{gcd}\left(F_{9}^{(1)}-\left(F_{1}-1\right), F_{10}^{(1)}+\left(F_{0}+1\right)\right) \\
= & \operatorname{gcd}\left(F_{8}^{(1)}+\left(F_{2}+1\right), F_{9}^{(1)}-\left(F_{1}-1\right)\right) \\
= & \operatorname{gcd}\left(F_{7}^{(1)}-\left(F_{3}-1\right), F_{8}^{(1)}+\left(F_{2}+1\right)\right) \\
= & \operatorname{gcd}\left(F_{4}^{(1)}+\left(F_{6}+1\right), F_{5}^{(1)}-\left(F_{5}-1\right)\right) \\
= & \operatorname{gcd}\left(2 F_{6}, F_{6}\right)=F_{6} .
\end{aligned}
$$

Indeed, $\operatorname{gcd}(88,232)=8$ which is the 6 -th number in the Fibonacci sequence.

When applying (6) we have an obvious consequence of Theorem 2, stated in Corollary 1.

Corollary 1. The greatest common divisor of the 4-tuple of hyperfibonacci numbers $F_{4 m-1}^{(1)}, F_{4 m-3}^{(1)}, F_{4 n-1}^{(1)}, F_{4 n-3}^{(1)}, m, n \in \mathbb{N}$ is equal to the $\operatorname{gcd}(2 m, 2 n)$-th Fibonacci number,

$$
\operatorname{gcd}\left(F_{4 m-1}^{(1)}, F_{4 m-3}^{(1)}, F_{4 n-1}^{(1)}, F_{4 n-3}^{(1)}\right)=F_{\operatorname{gcd}(2 m, 2 n)} .
$$

In Corollary 2 we list further periodicity in relatively prime pairs and the greatest common divisor, for hyperfibonacci numbers.

Corollary 2. For the hyperfibonacci sequence $\left(F_{n}^{(1)}\right)_{n \geq 0}$ we have

i) $\operatorname{gcd}\left(F_{6 n+4}^{(1)}, F_{6 n+5}^{(1)}\right)=1$, 
ii) $\operatorname{gcd}\left(F_{6 n+2}^{(1)}, F_{6 n+3}^{(1)}\right)=2$,

iii) $\operatorname{gcd}\left(F_{6 n+6}^{(1)}, F_{6 n+7}^{(1)}\right)=1$.

Proof. i) We employ recurrence relation (11) to get

$$
\begin{aligned}
& \operatorname{gcd}\left(F_{6 n+4}^{(1)}, F_{6 n+5}^{(1)}\right)=\operatorname{gcd}\left(F_{6 n+3}^{(1)}+1, F_{6 n+4}^{(1)}\right) \\
= & \operatorname{gcd}\left(F_{6 n+2}^{(1)}, F_{6 n+3}^{(1)}+1\right)=\operatorname{gcd}\left(F_{6 n+1}^{(1)}+2, F_{6 n+2}^{(1)}\right) \\
= & \operatorname{gcd}\left(F_{6 n}^{(1)}-1, F_{6 n+1}^{(1)}+2\right) \\
= & \operatorname{gcd}\left(F_{6 n-1}^{(1)}+\left(F_{4}+1\right), F_{6 n}^{(1)}-\left(F_{3}-1\right)\right) \\
= & \operatorname{gcd}\left(F_{6 n-2}^{(1)}-\left(F_{5}-1\right), F_{6 n+5}^{(1)}+\left(F_{4}+1\right)\right)
\end{aligned}
$$

When iteratively applying relation (11) and the basic properties of the gcd function we obtain

$$
\operatorname{gcd}\left(F_{6 n+4}^{(1)}, F_{6 n+5}^{(1)}\right)=\operatorname{gcd}\left(F_{3 n+1}^{(1)}-\left(F_{3 n+2}-1\right), F_{3 n+2}^{(1)}+\left(F_{3 n+1}+1\right)\right) .
$$

From the fact that the sum of the first $n$ numbers in Fibonacci sequence $\left(F_{n}\right)_{n \geq 0}$ is equal to $F_{n+2}-1$, we immediately have

$$
F_{n}^{(1)}=F_{n+2}-1 .
$$

We substitute (17) into r.h.s. of relation (16) to get

$$
\operatorname{gcd}\left(F_{6 n+4}^{(1)}, F_{6 n+5}^{(1)}\right)=\operatorname{gcd}\left(F_{3 n+1}, F_{3 n+4}\right) .
$$

Having in mind that

$$
\begin{aligned}
\operatorname{gcd}(3 n+1,3 n+4) & =\operatorname{gcd}(3 n+1,3) \\
& =1
\end{aligned}
$$

we finally have

$$
\begin{aligned}
\operatorname{gcd}\left(F_{6 n+4}^{(1)}, F_{6 n+5}^{(1)}\right) & =\operatorname{gcd}\left(F_{3 n+1}, F_{3 n+4}\right) \\
& =F_{\operatorname{gcd}(3 n+1,3 n+4)}=F_{1} \\
& =1 .
\end{aligned}
$$


Proof. ii) Once having equality

$$
\operatorname{gcd}\left(F_{6 n+2}^{(1)}, F_{6 n+3}^{(1)}\right)=\operatorname{gcd}\left(F_{3 n+3}, F_{3 n}\right)
$$

we get

$$
\begin{aligned}
\operatorname{gcd}(3 n+3,3 n) & =(3 n, 3) \\
& =3
\end{aligned}
$$

and finally

$$
\begin{aligned}
\operatorname{gcd}\left(F_{6 n+2}^{(1)}, F_{6 n+3}^{(1)}\right) & =\operatorname{gcd}\left(F_{3 n+3}, F_{3 n}\right) \\
& =F_{3}=2 .
\end{aligned}
$$

iii) Having in mind that

$$
\begin{aligned}
\operatorname{gcd}(3 n+2,3 n+5) & =\operatorname{gcd}(3 n+2,3) \\
& =\operatorname{gcd}(2,3)=1
\end{aligned}
$$

we obtain

$$
\begin{aligned}
\operatorname{gcd}\left(F_{6 n+6}^{(1)}, F_{6 n+7}^{(1)}\right) & =\operatorname{gcd}\left(F_{3 n+5}, F_{3 n+2}\right) \\
& =F_{\operatorname{gcd}(3 n+5,3 n+2)} \\
& =F_{1}=1
\end{aligned}
$$

which completes the proof.

We consider the alternative way of calculating $\operatorname{gcd}\left(F_{n}^{(1)}, F_{n+1}^{(1)}\right) . \operatorname{The} \operatorname{gcd}\left(F_{n}^{(1)}, F_{n+1}^{(1)}\right)$ can be written as

$$
\begin{aligned}
\operatorname{gcd}\left(F_{n}^{(1)}, F_{n+1}^{(1)}\right)= & \\
\operatorname{gcd}\left(F_{n+2}-1, F_{n+3}-1\right)= & \operatorname{gcd}\left(F_{n+2}-F_{-1}, F_{n+3}+F_{-2}\right)= \\
\operatorname{gcd}\left(F_{n+2}-F_{-1}, F_{n+1}+F_{0}\right)= & \operatorname{gcd}\left(F_{n}-F_{1}, F_{n+1}+F_{0}\right)= \\
\operatorname{gcd}\left(F_{n}-F_{1}, F_{n-1}+F_{2}\right)= & \operatorname{gcd}\left(F_{n-2}-F_{3}, F_{n-1}+F_{2}\right)= \\
\operatorname{gcd}\left(F_{n-2}-F_{3}, F_{n-3}+F_{4}\right)= & \operatorname{gcd}\left(F_{n-4}-F_{5}, F_{n-3}+F_{4}\right)=\ldots= \\
& \operatorname{gcd}\left(F_{n-2 k}-F_{2 k+1}, F_{n-2 k+1}+F_{2 k}\right)
\end{aligned}
$$

Now it follows: 
(a) $n=4 m, k=m, \operatorname{gcd}\left(F_{4 m}^{(1)}, F_{4 m+1}^{(1)}\right)=\operatorname{gcd}\left(F_{2 m}-F_{2 m+1}, F_{2 m+1}+F_{2 m}\right)=$ $\operatorname{gcd}\left(-F_{2 m-1}, F_{2 m+2}\right)=\operatorname{gcd}\left(F_{2 m-1}, F_{2 m+2}\right)=F_{\operatorname{gcd}(2 m-1,2 m+2)}=F_{\operatorname{gcd}(m+1,3)}=$ $F_{\operatorname{gcd}(n+1,3)}$

(b) $n=4 m+1, k=m, \operatorname{gcd}\left(F_{4 m+1}^{(1)}, F_{4 m+2}^{(1)}\right)=\operatorname{gcd}\left(F_{2 m+1}-F_{2 m+1}, F_{2 m+2}+F_{2 m}\right)=$ $\operatorname{gcd}\left(0, L_{2 m+1}\right)=L_{\frac{n+1}{2}}$

(c) $n=4 m+2, k=m, \operatorname{gcd}\left(F_{4 m+2}^{(1)}, F_{4 m+3}^{(1)}\right)=\operatorname{gcd}\left(F_{2 m+2}-F_{2 m+1}, F_{2 m+3}+F_{2 m}\right)=$ $\operatorname{gcd}\left(F_{2 m}, F_{2 m+3}+F_{2 m}\right)=\operatorname{gcd}\left(F_{2 m}, F_{2 m+3}\right)=F_{\operatorname{gcd}(2 m, 2 m+3)}=F_{\operatorname{gcd}(m, 3)}=F_{\operatorname{gcd}(n, 3)}$

(d) $n=4 m+3, k=m+1, \operatorname{gcd}\left(F_{4 m+3}^{(1)}, F_{4 m+4}^{(1)}\right)=\operatorname{gcd}\left(F_{2 m+1}-F_{2 m+3}, F_{2 m+2}+F_{2 m+2}\right)=$ $\operatorname{gcd}\left(-F_{2 m+2}, 2 F_{2 m+2}\right)=F_{2 m+2}=F_{\frac{n+1}{2}}$

\section{Congruences for $F_{p-1}^{(1)}, F_{p-2}^{(1)}$ and $F_{p-3}^{(1)}$}

Once having relation (17), we immediately obtain congruences for $F_{p-2}^{(1)}$, by substitution into (7). We present these congruences in the following Theorem 3 , where we also give more detailed proof.

Theorem 3. Let $p$ be an odd prime. Then for the hyperfibonacci sequence we have

$$
F_{p-2}^{(1)} \equiv\left(\frac{p}{5}\right)-1 \quad(\bmod p) .
$$

Proof. When applying the binomial theorem to the Binet's formula we get

$$
\begin{aligned}
F_{p-2}^{(1)} & =\frac{1}{\sqrt{5}}\left[\left(\frac{1+\sqrt{5}}{2}\right)^{p}-\left(\frac{1-\sqrt{5}}{2}\right)^{p}\right]-1 \\
& =\frac{1}{2^{p} \sqrt{5}} \sum_{k=0}^{p}\left(\begin{array}{l}
p \\
k
\end{array}\right)\left((\sqrt{5})^{k}-(-\sqrt{5})^{k}\right)-1 \\
& =\frac{1}{2^{p-1}} \sum_{k=0,2 \nmid k}^{p}\left(\begin{array}{l}
p \\
k
\end{array}\right) 5^{\frac{k-1}{2}}-1
\end{aligned}
$$

Having in mind an obvious fact that $p \mid\left(\begin{array}{l}p \\ k\end{array}\right), \quad k=1,2, \ldots, p-1$ we obtain

$$
1+2^{p-1} F_{p-2}^{(1)} \equiv 5^{\frac{p-1}{2}} \quad(\bmod p)
$$

and furthermore from the Euler's criterion

$$
1+2^{p-1} F_{p-2}^{(1)} \equiv\left(\frac{5}{p}\right) \quad(\bmod p) .
$$


In the similar fashion one can prove Theorem 4. Both congruences in Theorem 4 also follows by substitution of (17) into (8).

Theorem 4. Let $p$ be an odd prime. Then for the hyperfibonacci sequence we have

$$
F_{p-3}^{(1)} \equiv \frac{-1-\left(\frac{p}{5}\right)}{2} \quad(\bmod p)
$$

and

$$
F_{p-1}^{(1)} \equiv \frac{-1+\left(\frac{p}{5}\right)}{2} \quad(\bmod p) .
$$

According to the quadratic reciprocity theorem we get equality

$$
\left(\frac{5}{p}\right)=\left(\frac{p}{5}\right)
$$

which gives

$$
\left(\frac{p}{5}\right)=\left\{\begin{array}{lll}
1 & \text { if } p \equiv \pm 1 & (\bmod 5) \\
-1 & \text { if } p \equiv \pm 2 & (\bmod 5)
\end{array}\right.
$$

when we employ basic properties of the Legendre symbol. Now we have immediate consequences of Theorems 3 and 4 . Corollary 3 follows from the congruence (20) while Corollary 4 follows from congruences (19) and (21).

Corollary 3. Let $p$ be a prime such that $p \equiv \pm 2(\bmod 5)$. Then $p \mid F_{p-3}^{(1)}$.

Corollary 4. Let $p$ be a prime such that $p \equiv \pm 1(\bmod 5)$. Then $p \mid F_{p-2}^{(1)}$ and $p \mid F_{p-1}^{(1)}$.

\section{Concluding remarks and open questions}

We believe that results obtained in this paper can be extended to other families of strong divisibility sequences. It would be of interest to find periodicity of gcd for other generation of hyperfibonacci numbers and possibly to give statements in full generality. There are also a few other generalization of recursive sequences of numbers $([9,12,15])$ and it is of interest to see an extension of property (2) within these sequences. Some further generalizations and extensions of these sequences would be of interest as well. 


\section{Acknowledgment}

The authors thank the referee for careful reading, valuable help and valuable suggestions that improved the final version of the paper.

\section{References}

[1] A.T. Benjamin, J.J. Quinn, Proofs that Really Count, The Mathematical Association of America, 2003.

[2] J.P. Bézivin, A. Pethő, A.J. van der Poorten, A full characterization of divisibility sequences, Am. J. Math. 112 (6) (1990), 985-1001.

[3] N. N. Cao and F. Z. Zhao, Some properties of Hyperfibonacci and Hyperlucas Numbers, J. Integer Seq. 13 (2010), Article 10.8.8.

[4] G. Cornelissen, J. Reynolds, Matrix divisibility sequences, Acta Arith., 156 (2012), 177-188.

[5] L. Cristea, I. Martinjak, I. Urbiha, Hyperfibonacci Sequences and Polytopic Numbers, J. Integer Seq., 19/7 (2016), Article 16.7.6, 13pp.

[6] B. Gezer, O. Bizim, Squares in elliptic divisibility sequences, Acta Arith., 144.2 (2010), 125-134.

[7] M. Glunčić, I. Martinjak, A Class of $S$-Restricted Compositions, Int. Journal of Number Theory, to appear, https://doi.org/10.1142/S1793042119500180

[8] A. Dil and I. Mezö, A symmetric algorithm for hyperharmonic and Fibonacci numbers, Appl. Math. Comput., 206 (2008), 942-951.

[9] T. Došlić, I. Martinjak, R. Škrekovski, Total Positivity of Toeplitz Matrices of Recursive Hypersequences, preprint

[10] K. Górnisiewicz, New Examples of Divisibility Sequences, Integers 16 (2006), \#A56, 5pp.

[11] P. Ingram, Elliptic divisibility sequences over certain curves, J. Number Theory 123 (2) (2007), 473-486. 
[12] C. Krattenthaler and A.M. Oller-Marcén, A Determinant of Generalized Fibonacci Numbers, J. Combin. Number Theory 5(2) (2003), article 2 1-7.

[13] R. Liu and F. Zhao, On the sums of reciprocal Hyperfibonacci numbers and Hyperlucas numbers, J. Integer Seq. 15 (2012), Article 12.4.5.

[14] I. Martinjak, I. Urbiha, A New Generalized Cassini Determinant, Colloq. Math. 145 (2), 2016, 209-218

[15] E. Miles, Generalized Fibonacci numbers and related matrices, Amer. Math. Monthly 67 (1960), 745-752.

[16] J. H. Silverman, Divisibility Sequences and Powers of Algebraic Integers, Documenta Math., Extra Volume Coates (2006), 711-727.

[17] J.H. Silverman, Generalized greatest common divisors, divisibility sequences, and Vojta's conjecture for blowups, Monatsh. Math., 145 (2015), 333-350.

[18] J.H. Silverman, Common divisors of elliptic divisibility sequences over function fields, Manuscripta Math., 114 (2004), 432-446.

[19] Z. H. Sun, Z. W. Sun, Fibonacci numbers and Fermat's last theorem, Acta Arith., 60 (4) (1992), 371-388.

[20] L. Zheng, R. Liu, and F. Zhao, On the log-concavity of the hyperfibonacci numbers and the hyperlucas numbers, J. Integer Seq., 17 (2014), Article 14.1.4 